\title{
Finite-Time Fault Detection for Large-Scale Networked Systems with Randomly Occurring Nonlinearity and Fault
}

\author{
Yong Zhang, ${ }^{1}$ Huajing Fang, ${ }^{2}$ and Zhenxing Liu ${ }^{1}$ \\ ${ }^{1}$ School of Information Science and Engineering, Wuhan University of Science and Technology, Wuhan 430081, China \\ ${ }^{2}$ School of Automation, Huazhong University of Science and Technology, Wuhan 430074, China \\ Correspondence should be addressed to Yong Zhang; zhangyong77@wust.edu.cn
}

Received 14 January 2014; Accepted 15 March 2014; Published 27 April 2014

Academic Editor: Housheng Su

Copyright (c) 2014 Yong Zhang et al. This is an open access article distributed under the Creative Commons Attribution License, which permits unrestricted use, distribution, and reproduction in any medium, provided the original work is properly cited.

\begin{abstract}
The finite-time fault detection problem is investigated for a class of nonlinear quantized large-scale networked systems with randomly occurring nonlinearities and faults. A nonlinear Markovian jump system model with partially unknown transition probabilities is employed to describe this Makov data assignment pattern. Based on obtained model, in finite-time stable framework, the desired mode-dependent fault detection filters are constructed such that the augmented error systems are finitetime stochastically stable with $H_{\infty}$ attenuation level. Especially, the sufficient conclusions provide quantitative relationship between network characteristic, quantization level, and finite-time system parameter with finite-time fault detection performance. The effectiveness of the proposed methods is demonstrated by simulation examples.
\end{abstract}

\section{Introduction}

The past decade has witnessed an ever increasing research interest in networked systems (NSs) due to their advantages in many aspects such as low cost, simple installation and maintenance, increased system agility, reduced system wiring, and high reliability. For large-scale NSs, multiple sensors and actuators are connected to the central control and fault detection station through communication medium. Actually, the introduction of communication network inevitably brings communication constraints to the systems analysis and synthesis. Especially, network-induced delay, packet dropout, and signal quantization have significant effect on the performance and stability, even fault of NSs [1-9].

Fault detection has been an active research field over the past decades because of the ever increasing demand for higher performance, higher safety, and reliability standard [10-12]. Recently, there are increasing interests on fault detection (FD) of networked systems. However, compared with the rich results in control and stability analysis of networked systems, only a limited number of contributions about FD have been found [13]. To deal with the FD of nonlinear networked systems (NCSs), [14] study T-S fuzzy model based fault detection for NCSs with stochastic mixed time delays and successive packet dropouts. In [15], an FD framework for a class of nonlinear NCSs via a shared communication medium has been proposed. In addition, the robust fault detection problem is studied in [16] for a class of NSs with unknown input, multiple state delays, and data missing. [17] study robust fault detection of NSs with delay distribution characterization.

For networked systems, stability analysis may be one of the most important research attention. Even almost all existing stability results are Lyapunov stability, actually, finitetime stability (FTS) [18] is a more practical concept which is utilized to study the behavior of the system within a finite time interval. Markovian jump systems (MJSs) are said to be stochastically finite-time stable if once we fix a finite-time interval, its state remains within prescribed bounds during this time interval. Obviously, MJSs may be not Lyapunov stochastically stable but finite-time stable. For large-scale networked systems, the nonlinear may be random due to stochastic change from network-induced phenomenon, which give rise to the so-called randomly occurring nonlinearities (RONs) [19]. Actually, compared 
to deterministic fault of networked systems [13-17], faults may also occur in a probabilistic way and they arerandomly changeable in terms of their types and/or intensity. To the best of the authors' knowledge, up to now, almost no attention has been paid to the study of finite-time fault detection for nonlinear multiple channels data transmission networked systems with RONs and randomly occurring faults (ROFs); the main purpose of this paper is to shorten such a gap.

The main contributions of this paper are summarized as follows. (1) A Markovian jump system model with partially unknown transition probabilities is proposed to describe the multiple channels data transmission networked systems with channel-dependent measurement quantization; (2) based on the obtained model, by utilizing observer-based fault detection filter as residual generator, finite-time fault detection of large-scale networked systems is formulated as nonlinear finite-time $H_{\infty}$ attenuation problem; and (3) by means of linear matrix inequalities (LMIs) method, sufficient conditions of finite-time stochastic stability are obtained and $H_{\infty}$ attenuation level is guaranteed, and the explicit expression of the desired mode-dependent fault detection filters is also derived, which establish the quantitative relationship between quantization level and finite-time system parameters with fault detection performance. Especially, fault detection of traditional Markovian jump systems (known transition probability) and switched systems (unknown transition probability) with Lyapunov asymptotic stability (assuming finitetime system parameter $\sigma=1$ ) can be contained as its special case. Numerical simulations are utilized to demonstrate the effectiveness of the presented methods.

Notation. Throughout the paper, the superscripts " -1 " and " $T$ " stand for the inverse and transpose of a matrix, respectively. $\mathbb{R}^{n}$ denotes the $n$-dimensional Euclidean space and $\|\cdot\|$ refers to Euclidean norm for vectors. $P>0(\geq 0)$ means that $P$ is a real symmetric positive definite (semidefinite) matrix. $\mathbf{E}\{x\}$ is the expectation of the stochastic variable $x$. $\operatorname{Prob}\{\cdot\}$ means the occurrence probability of event “.”. I and 0 represent identity matrix and zero matrix; we utilize asterisk (*) to represent a term that is induced by symmetry and $\operatorname{diag}\{\ldots\}$ stands for a block diagonal matrix.

\section{Problem Formulation}

Consider the discrete-time NSs with the following nonlinear system model:

$$
\begin{aligned}
& x(k+1)= A x(k)+\alpha(k) N_{1} g(x(k)) \\
&+B_{1} d(k)+\beta(k) B_{2} f(k) \\
& y(k)=C x(k)
\end{aligned}
$$

where $x(k) \in \mathbb{R}^{n_{x}}$ is the state vector, $y(k) \in \mathbb{R}^{n_{y}}$ is the measurement output, $d(k) \in \mathbb{R}^{n_{d}}$ is the disturbance input vector, and $f(k) \in \mathbb{R}^{n_{f}}$ is the fault vector to be detected. $A$, $B_{1}, B_{2}, N_{1}$, and $C$ are known real matrices with appropriate dimension. The nonlinear function $g(x(k))$ satisfies $g(0)=0$ and the following condition:

$$
g^{T}(x(k)) g(x(k)) \leq \delta x^{T}(k) G^{T} G x(k),
$$

where $\delta>0$ is the bounding parameters on the nonlinear function $g(x(k))$; $G$ is known real constant matrix.

Random variables $\alpha(k) \in \mathbb{R}$ and $\beta(k) \in \mathbb{R}$ are utilized to account for the phenomena of randomly occurred nonlinearities and faults, which are assumed to be independent from each other and take values of 0 and 1 with

$$
\begin{aligned}
& \operatorname{Prob}(\alpha(k)=1)=\mathbf{E}\{\alpha(k)\}=\bar{\alpha} ; \\
& \operatorname{Prob}(\beta(k)=1)=\mathbf{E}\{\beta(k)\}=\bar{\beta},
\end{aligned}
$$

where $\bar{\alpha} \in[0,1]$ and $\bar{\beta} \in[0,1]$ are known constants. For large-scale complex networked systems, the nonlinearities and faults may be subject to random changes in environmental circumstances, for instance, network-induced random failures and repairs of components, sudden environmental disturbances, and so forth. Therefore, both the nonlinearities and faults may occur in a probabilistic way with certain types and intensity, which is particularly true in a networked environment.

In this paper, before accessing the observer, output signal $y(k)$ will be quantized by quantizer $q(\cdot)$ and quantized signal $\widetilde{y}(k)$ can be expressed as

$$
\tilde{y}(k)=q(y(k))=\left[q_{1}\left(y_{1}(k)\right), \ldots, q_{n_{y}}\left(y_{n_{y}}(k)\right)\right]^{T} .
$$

If quantizer $q(\cdot)$ is assumed to be logarithmic type, the set of quantization levels $q_{j}(\cdot)\left(1 \leq j \leq n_{y}\right)$ is described by $\mathfrak{\Im}=$ $\left\{ \pm u_{i}^{(j)}, u_{i}^{(j)}=\chi_{j}^{i} u_{0}^{(j)}, i=0, \pm 1, \pm 2, \ldots\right\} \bigcup\{0\}, 0<\chi_{j}<1, u_{0}^{(j)}>$ 0 . Each quantization level corresponds to a segment such that the quantizer maps the whole segment to this quantization level. In addition, these segments form a partition of $\mathbb{R}$; that is, they are disjoint and their union equals $\mathbb{R}$. The logarithmic quantizer $q_{j}(\cdot)$ is defined as

$$
\begin{aligned}
& q_{j}\left(y_{j}(k)\right) \\
& = \begin{cases}u_{i}^{(j)}, & \frac{1}{1+\bar{\delta}_{j}} u_{i}^{(j)}<y_{j}(k) \leq \frac{1}{1-\bar{\delta}_{j}} u_{i}^{(j)} \\
0, & y_{j}(k)=0 \\
-q_{j}\left(-y_{j}(k)\right), & y_{j}(k)<0\end{cases}
\end{aligned}
$$

where $\bar{\delta}_{j}=\left(1-\chi_{j}\right) /\left(1+\chi_{j}\right)$. Similar to [20], we have the expression of $q_{j}\left(y_{j}(k)\right)=\left(1+\Delta_{j}(k)\right) y_{j}(k)$ with $\left|\Delta_{j}(k)\right| \leq \bar{\delta}_{j}$. Defining $\Delta(k)=\operatorname{diag}\left\{\Delta_{1}(k), \ldots, \Delta_{n_{y}}(k)\right\}$, the measurements after quantization have the following form:

$$
\begin{aligned}
\tilde{y}(k)= & (I+\Delta(k)) y(k) \\
= & (I+\Delta(k)) C x(k)+(I+\Delta(k)) D_{1} d(k) \\
& +(I+\Delta(k)) D_{2} f(k) .
\end{aligned}
$$


Actually, the quantized effect can be transformed into sector bound uncertainties. By defining $\bar{\Delta}=\operatorname{diag}\left\{\delta_{1}, \ldots, \delta_{n_{y}}\right\}$ and $F(k)=\Delta(k) \bar{\Delta}^{-1}$, we obtain an unknown real-valued time-varying matrix $F(k)$ satisfying $F(k) F^{T}(k) \leq I$.

In this paper, two-valued function $\theta_{l}(k): Z \rightarrow\{0,1\}(l=$ $\left.1, \ldots, n_{y}\right)$ is used to describe the $l$ th channels transmission status in sampling time $k$, where 1 means successful data transmission and 0 means data loss. Specifically, only corresponding data packet access the communication medium, that is, $\theta_{l}(k)=1$, the quantized output $q_{l}\left(y_{l}(k)\right)$ to observer of $l$ th channels is available. Otherwise, when $\theta_{l}(k)=0$, the output of $l$ th channels will be zero by the observer and $q_{l}\left(y_{l}(k)\right)$ will be ignored due to its being unavailable. If we regard $\bar{y}_{l}(k)$ as the $l$ th channels signal received by the observer, we describe the transmission dynamics of $l$ th channels as:

$$
\bar{y}_{l}(k)=\theta_{l}(k) q_{l}\left(y_{l}(k)\right) \quad\left(l=1, \ldots, n_{y}\right) .
$$

In the need of investigation, we define transmission matrix as $M_{n_{y}} \triangleq\left\{M_{n_{y}}^{0,1}, M_{n_{y}}^{1,1}, \ldots, M_{n_{y}}^{1, n_{y}}, \ldots, M_{n_{y}}^{n_{y}-1,1}, \ldots\right.$, $\left.M_{n_{y}}^{n_{y}-1, n_{y}}, M_{n_{y}}^{n_{y}, 1}\right\}$, and matrix $M_{n_{y}}^{s, t}=\operatorname{diag}\left\{\theta_{1}(k), \ldots, \theta_{n_{y}}(k)\right\}$ can be expressed in the following form:

$$
\begin{aligned}
M_{n_{y}}^{0,1} \triangleq \operatorname{diag}\{\underbrace{0, \ldots, 0}_{n_{y}}\}, \\
M_{n_{y}}^{1,1} \triangleq \operatorname{diag}\{1, \underbrace{0, \ldots, 0}_{n_{y}-1}\}, \ldots, \\
M_{n_{y}}^{1, n_{y}} \triangleq \operatorname{diag}\{\underbrace{0, \ldots, 0}_{n_{y}-1}, 1\}, \ldots, \\
M_{n_{y}}^{n_{y}-1,1} \triangleq \operatorname{diag}\{\underbrace{1, \ldots, 1}_{n_{y}-1}, 0\}, \ldots, \\
M_{n_{y}}^{n_{y}-1, n_{y}} \triangleq \operatorname{diag}\{0, \underbrace{0, \ldots, 1}_{n_{y}-1}\}, \\
M_{n_{y}}^{n_{y}, 1} \triangleq \operatorname{diag}\{\underbrace{1, \ldots, 1}_{n_{y}}\} .
\end{aligned}
$$

According to above discussion, we achieve the quantized output dynamics as

$$
\bar{y}(k)=M_{n_{y}}^{\mu(k)} \tilde{y}(k)=M_{n_{y}}^{\mu(k)} q(\widetilde{y}(k)),
$$

where $M_{n_{y}}^{\mu(k)} \in M_{n_{y}}$. Let $\mu(k)$ be a Markov chain taking values in a finite state space $\Re=\left\{1,2, \ldots, 2^{n_{y}}\right\}$ with transition probability matrix $\Lambda=\left(\rho_{i j}\right)$ given by

$$
\rho_{i j}=\operatorname{Prob}(\mu(k+1)=j \mid \mu(k)=i),
$$

where $\rho_{i j} \geq 0, \forall i, j \in \Re$, and $\sum_{j=1}^{2^{n_{y}}} \rho_{i j}=1$. It is suitable that we assume that Markov chain $\mu(k)$ is independent of the stochastic variables $\alpha(k)$ and $\beta(k)$.

In this paper, the following mode-dependent observerbased fault detection filter is constructed as a residual generator:

$$
\begin{gathered}
\widehat{x}(k+1)=A \widehat{x}(k)+L_{\mu(k)}(\bar{y}(k)-\hat{y}(k)), \\
\hat{y}(k)=C \widehat{x}(k), \\
r(k)=V_{\mu(k)}(\bar{y}(k)-\hat{y}(k)),
\end{gathered}
$$

where $\widehat{x}(k) \in \mathbb{R}^{n_{\widehat{x}}}$ and $\widehat{y}(k) \in \mathbb{R}^{n_{\hat{y}}}$ represent the state and output estimation vectors, respectively. $r(k)$ is the residual signal. FDF parameters are the observer gain matrices $L_{\mu(k)}$ and residual weighting matrices $V_{\mu(k)}(\forall \mu(k) \in \Re)$. Observer with above structure is assumed to jump synchronously with the modes in (9), which is hereby mode-dependent.

Let the estimation error be $e_{1}(k)=x(k)-\widehat{x}(k)$, then error systems can be obtained by combining (1), (9), and (11):

$$
\begin{aligned}
e_{1}(k+1)= & \left(A-L_{\mu(k)} C\right) e_{1}(k)-L_{\mu(k)} \\
& \times\left[M_{p}^{\mu(k)}(I+\Delta(k)) C-C\right] x(k) \\
& +\alpha(k) N_{1} g(x(k))+B_{1} d(k)+\beta(k) B_{2} f(k) .
\end{aligned}
$$

By setting $r_{e}(k)=r(k)-f(k), e(k)=\left[x^{T}(k), e_{1}^{T}(k)\right]^{T}$, $w(k)=\left[d^{T}(k), f^{T}(k)\right]^{T}, g(e(k))=\left[g^{T}(x(k)), g^{T}\left(e_{1}(k)\right)\right]^{T}$ and integrating (1) and (12), we obtain the following augmented error systems:

$$
\begin{gathered}
e(k+1)=\bar{A}_{\mu(k)} e(k)+\alpha(k) \bar{N}_{1} H g_{1}(e(k)) \\
+\left[\bar{B}_{1}+\beta(k) \bar{B}_{2}\right] w(k) \\
r_{e}(k)=\bar{C}_{\mu(k)} e(k)+\beta(k) \bar{D} w(k),
\end{gathered}
$$

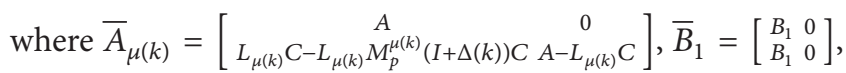
$\bar{B}_{2}=\left[\begin{array}{ll}0 & B_{2} \\ 0 & B_{2}\end{array}\right] \bar{N}_{1}=\left[\begin{array}{l}N_{1} \\ N_{1}\end{array}\right], \bar{C}_{\mu(k)}=\left[V_{\mu(k)} M_{p}^{\mu(k)}(I+\Delta(k)) C-\right.$ $\left.V_{\mu(k)} C, V_{\mu(k)} C\right], D=\left[\begin{array}{ll}0 & -I\end{array}\right]$, and $H=\left[\begin{array}{ll}I & 0\end{array}\right] ; r_{e}(k)$ is the residual error which contains the stochastic fault information of occurrence time and location. In addition, the transition probabilities of jumping process $\{\mu(k), k \geq 0\}$ are assumed to be partly accessed; that is, some elements are unknown. For notation clarity, we denote $\mathfrak{R}=\mathfrak{R}_{K}^{i}+\mathfrak{R}_{U K}^{i}(\forall i \in \mathfrak{R})$ with $\mathfrak{R}_{K}^{i} \triangleq\left\{j: \rho_{i j}\right.$ is known $\}$ and $\mathfrak{R}_{U K}^{i} \triangleq\left\{j: \rho_{i j}\right.$ is unknown $\}$.

Now, to present the main objective of this paper more precisely, we need the following finite-time stochastic stability definition for augmented error systems (13), which are essential for the later development. 
Definition 1. Augmented error systems (13) are said to be finite-time stochastically stable with respect to $\left(c_{1}, c_{2}, R, N\right)$ for $w(k)=0$ and every initial condition $e(0)$, where $R>0$ are positive define matrix, $0<c_{1}<c_{2}$ and $N \in \mathbb{Z}$, if

$$
e^{T}(0) \operatorname{Re}(0) \leq c_{1} \Longrightarrow \mathbf{E}\left[e^{T}(k) \operatorname{Re}(k)\right]<c_{2} .
$$

The purpose of this paper is to design mode-dependent observer-based fault detection parameters $L_{\mu(k)}$ and $V_{\mu(k)}$ such that augmented error systems (13) are finite-time stochastically stable; under zero-initial condition, for any nonzero $w(k)$, we have

$$
\sum_{k=0}^{\infty} \mathbf{E}\left\{\left\|r_{e}(k)\right\|^{2}\right\}<\gamma^{2} \sum_{k=0}^{\infty}\|w(k)\|^{2} .
$$

In order to detect the faults, the widely adopted approach is to choose an appropriate threshold $J_{\text {th }}$ and residual evolution function $J\left(r_{e}\right)$ as

$$
\begin{gathered}
J\left(r_{e}\right)=\mathbf{E}\left\{\sum_{k=k_{0}}^{k=k_{0}+\kappa} r_{e}^{T}(k) r_{e}(k)\right\}^{1 / 2}, \\
J_{\text {th }}=\sup _{d(k) \in \ell_{2}, f(k)=0} J\left(r_{e}\right),
\end{gathered}
$$

where $k_{0}$ denotes the initial evaluation time instant and $\kappa$ denotes the evaluation time steps.
Based on the threshold, the occurrence of fault can be detected by comparing $J_{\text {th }}$ and $J\left(r_{e}\right)$ according to the following test:

$$
\begin{gathered}
J\left(r_{e}\right) \geq J_{\text {th }} \Longrightarrow \text { alarm for fault } \\
J\left(r_{e}\right)<J_{\text {th }} \Longrightarrow \text { no fault. }
\end{gathered}
$$

\section{Main Results}

The following theorem provides a sufficient condition under which the augmented error systems (13) are finite-time stochastically stable and the residual estimation error $r_{e}(k)$ satisfies the $H_{\infty}$ criterion (15) under zero-initial condition.

Theorem 2. Augmented error systems (13) are finite-time stochastically stable with respect to $\left(c_{1}, c_{2}, R_{i}, N\right)$ and satisfy $H_{\infty}$ performance level (15), if there exist positive define matrices $P_{i}>0(i \in \mathfrak{R})$, scalars $\varepsilon_{s}>0, \bar{\sigma} \geq 1$, and $\gamma>0$ such that the following matrix inequalities hold:

$$
\begin{array}{ccc}
{\left[\begin{array}{ccc}
\Xi_{11} & \Xi_{12}^{T} & \Xi_{13}^{T} \Xi_{22} \\
* & \operatorname{diag}\{I, I\} & 0 \\
* & * & \Xi_{22}
\end{array}\right]<0,} \\
\frac{c_{1}^{2}}{\frac{\lambda}{\lambda}}<\frac{c_{2}^{2}}{\bar{\sigma}^{N} \bar{\lambda}},
\end{array}
$$

where

$$
\begin{aligned}
& \Xi_{12}^{T}=\left[\begin{array}{cc}
\sqrt{\bar{\beta}} \bar{C}_{i}^{T} & \sqrt{1-\bar{\beta}} \bar{C}_{i}^{T} \\
0 & 0 \\
\sqrt{\bar{\beta}} \bar{D}^{T} & 0
\end{array}\right], \\
& \Xi_{13}^{T}=\left[\begin{array}{ccc}
0 & \sqrt{1-\bar{\alpha} \bar{A}_{i}^{T}} & \sqrt{\bar{\alpha}} \bar{A}_{i}^{T} \\
0 & 0 & \sqrt{\bar{\alpha}} \bar{N}_{1}^{T} \\
\sqrt{\bar{\beta}(1-\bar{\beta})} \bar{B}_{2}^{T} & \sqrt{1-\bar{\alpha}}\left(\bar{B}_{2}^{T}+\bar{\beta} \bar{B}_{2}^{T}\right) & \sqrt{\bar{\alpha}}\left(\bar{B}_{2}^{T}+\bar{\beta} \bar{B}_{2}^{T}\right)
\end{array}\right], \\
& \Xi_{11}=\operatorname{diag}\left\{-\lambda P_{i}-\varepsilon_{1} \delta H^{T} G^{T} G H,-\varepsilon_{1} I,-\gamma^{2} I\right\}, \\
& \Xi_{22}=\operatorname{diag}\left\{-\Upsilon_{j},-\Upsilon_{j},-\Upsilon_{j}\right\}, \quad \Upsilon_{j} \triangleq \begin{cases}\frac{1}{\rho_{K}^{i}} P_{K}^{i}, & \forall j \in \mathfrak{R}_{K}^{i} \\
P_{j}, & \forall j \in \mathfrak{R}_{U K}^{i},\end{cases} \\
& P_{K}^{i}=\sum_{j \in \Re_{K}^{i}} \rho_{i j} P_{j}, \quad \rho_{K}^{i}=\sum_{j \in \Re_{K}^{i}} \rho_{i j}, \quad \widetilde{P}_{i}=R^{-1 / 2} P_{i} R^{-1 / 2}, \\
& \bar{\lambda}=\max _{i \in \mathfrak{R}} \lambda_{\max }\left(\widetilde{P}_{i}\right), \quad \underline{\lambda}=\min _{i \in \mathfrak{R}} \lambda_{\min }\left(\widetilde{P}_{i}\right) .
\end{aligned}
$$


Proof. For augmented error systems (13) with $w(k)=0$, we construct stochastic Lyapunov function as

$$
V(e(k), \mu(k))=e^{T}(k) P_{\mu(k)} e(k),
$$

where $P_{\mu(k)}>0(\mu(k) \in \Re)$. If we denote $\mu(k)=i$, then $P_{\mu(k)}$ is $P_{i}$. So, along the solution of (13), we obtain

$$
\begin{aligned}
\mathbf{E}[ & \Delta V(e(k))] \\
& =\mathbf{E}[V(e(k+1), k+1 \mid e(k), \mu(k))]-\bar{\sigma} V(e(k)) \\
& =e^{T}(k+1) \sum_{j \in \Re} \rho_{i j} P_{j} e(k+1)-\bar{\sigma} e^{T}(k) P_{i} e(k) \\
& =e^{T}(k+1)\left[\sum_{j \in \Re_{K}^{i}} \rho_{i j} P_{j}+\sum_{j \in \Re_{U K}^{i}} \rho_{i j} P_{j}\right] e(k+1) \\
& -\bar{\sigma} e^{T}(k)\left[\sum_{j \in \Re_{K}^{i}} \rho_{i j}+\sum_{j \in \Re_{U K}^{i}} \rho_{i j}\right] P_{i} e(k)
\end{aligned}
$$$$
\begin{gathered}
\Xi_{1}^{i}=\left[\begin{array}{cc}
\bar{A}_{i}^{T} P_{K}^{i} \bar{A}_{i}-\bar{\sigma} \rho_{K}^{i} P_{i}-\varepsilon_{1} \delta \rho_{K}^{i} H^{T} G^{T} G H & \bar{\alpha} \bar{A}_{i}^{T} P_{K}^{i} \bar{N}_{1} \\
* & \bar{\alpha} \bar{N}_{1}^{T} P_{K}^{i} \bar{N}_{1}-\varepsilon_{1} \rho_{K}^{i} I
\end{array}\right], \\
\Xi_{2}^{i}=\left[\begin{array}{cc}
\bar{A}_{i}^{T} P_{j} \bar{A}_{i}-\lambda P_{i}-\varepsilon_{1} \delta H^{T} G^{T} G H & \bar{\alpha} \bar{A}_{i}^{T} P_{j} \bar{N} \\
* & \bar{\alpha} \bar{N}^{T} P_{j} \bar{N}-\varepsilon_{1} I
\end{array}\right] .
\end{gathered}
$$

$$
\begin{aligned}
= & e^{T}(k+1) P_{K}^{i} e(k+1)-\bar{\sigma} e^{T}(k) \rho_{K}^{i} e(k) \\
& +\sum_{j \in \Re_{U K}^{i}} \rho_{i j}\left[e^{T}(k+1) P_{j} e(k+1)-\bar{\sigma} e^{T}(k) P_{i} e(k)\right] .
\end{aligned}
$$

For any scalar $\varepsilon_{1}>0$, it follows readily from (2) that

$\varepsilon_{1}\left(\delta e^{T}(k) H^{T} G^{T} G H e(k)-g^{T}(e(k)) H^{T} H g(e(k))\right) \geq 0$.

Combining (13) and (22)-(23) and denoting $\xi(k)=$ $\left[e^{T}(k), g^{T}(e(k)) H^{T}\right]^{T}$ lead to

$$
\mathbf{E}[\Delta V(\mathfrak{I}(k), k)]=\xi^{T}(k)\left\{\Xi_{1}^{i}+\sum_{j \in \Re_{U K}^{i}} \rho_{i j} \Xi_{2}^{i}\right\} \xi(k),
$$

where
By Schur complement, (18) implies $\Xi_{1}^{i}<0$ and $\Xi_{2}^{i}<0$, which means that

$$
\mathbf{E}[V(\xi(k+1))]<\bar{\sigma} V(\xi(k)) .
$$

Applying above inequality interactively, we obtain

$$
\mathbf{E}[V(\xi(k))]<\bar{\sigma}^{k} V(\xi(0)), \quad k=1, \ldots, N .
$$

Now, letting $\widetilde{P}_{i}=R^{-1 / 2} P_{i} R^{-1 / 2}$ and using the fact that $\bar{\sigma} \geq$ 1 , we have

$$
\begin{aligned}
\bar{\sigma}^{k} V(\xi(0), \mu(0)) & =\bar{\sigma}^{k} \xi^{T}(0) P_{i} \xi(0) \\
& \leq \bar{\sigma}^{k} \lambda_{\max }\left(\widetilde{P}_{i}\right) \xi^{T}(0) R \xi(0) \\
& \leq \bar{\sigma}^{N} \bar{\lambda}\left(\widetilde{P}_{i}\right) c_{1}^{2} .
\end{aligned}
$$

Furthermore, according to the following fact,

$$
\begin{aligned}
\mathbf{E}[V(\xi(k))] & =\mathbf{E}\left[\xi^{T}(k) P_{i} \xi(k)\right] \\
& =\mathbf{E}\left[\xi^{T}(k) R^{1 / 2} \widetilde{P}_{i} R^{1 / 2} \xi(k)\right] \\
& \geq \lambda_{\min }\left(\widetilde{P}_{i}\right) \xi^{T}(k) R \xi(k) \geq \underline{\lambda} \xi^{T}(k) R \xi(k) .
\end{aligned}
$$

Putting (27) and (29) together may yield

$$
\mathbf{E}\left[\xi^{T}(k) R \xi(k)\right] \leq \frac{\bar{\sigma}^{N}}{\underline{\lambda}} \bar{\lambda} c_{1}^{2} .
$$

Thus, augmented error systems (13) are finite-time stochastically stable from Definition 1.

On the other hand, under zero initial condition, for $w(k) \neq 0$ in (13), we consider the following performance index:

$$
J_{N}=\mathbf{E} \sum_{k=0}^{N}\left[r_{e}^{T}(k) r_{e}(k)-\gamma w^{T}(k) w(k)\right]
$$

$$
\text { Let } \zeta(k)=\left[\xi^{T}(k), w^{T}(k)\right]^{T} \text {, we have }
$$

$$
\begin{aligned}
J_{N}= & \mathbf{E} \sum_{k=0}^{N}\left[r_{e}^{T}(k) r_{e}(k)-\gamma^{2} w^{T}(k) w(k)+\Delta V(\mathfrak{I}(k))\right] \\
& -\mathbf{E} V(N+1) \\
\leq & \mathbf{E} \sum_{k=0}^{N}\left[r_{e}^{T}(k) r_{e}(k)-\gamma^{2} w^{T}(k) w(k)+\Delta V(\mathfrak{I}(k))\right] \\
= & \sum_{k=0}^{N} \zeta^{T}(k)\left[\bar{\Xi}_{1}^{i}+\sum_{j \in \Re_{U K}^{i}} \rho_{i j} \bar{\Xi}_{2}^{i}\right] \zeta(k),
\end{aligned}
$$


where

$$
\begin{gathered}
\bar{\Xi}_{1}^{i}=\left[\begin{array}{ll}
\Xi_{1}^{i} & \Omega_{1}^{i} \\
* & \Omega_{2}^{i}
\end{array}\right], \\
\Omega_{1}^{i}=\left[\bar{B}_{1}^{T} P_{K}^{i} \bar{A}_{i}+\bar{\beta} \bar{B}_{2}^{T} P_{K}^{i} \bar{A}_{i}+\rho_{K}^{i} \bar{\beta} \bar{C}_{i}^{T} \bar{D} \bar{\alpha}_{1}^{T} P_{K}^{i} \bar{N}_{1}+\bar{\alpha} \bar{\beta} \bar{B}_{2}^{T} P_{K}^{i} \bar{N}_{1}\right]^{T}, \\
\bar{\Xi}_{2}^{i}=\left[\begin{array}{cc}
\Xi_{2}^{i} & \Omega_{3}^{i} \\
* & \Omega_{4}^{i}
\end{array}\right], \\
\Omega_{2}^{i}=\bar{B}_{1}^{T} P_{K}^{i} \bar{B}_{1}+2 \bar{\beta} \bar{B}_{1}^{T} P_{K}^{i} \bar{B}_{2}+\bar{\beta}_{2}^{T} P_{K}^{i} \bar{B}_{2}+\bar{\beta}_{K}^{i} \bar{D}^{T} \bar{D}-\gamma^{2} \rho_{K}^{i} I, \\
\Omega_{3}^{i}=\left[\bar{B}_{1}^{T} P_{j} \bar{A}_{i}+\bar{\beta} \bar{B}_{2}^{T} P_{j} \bar{A}_{i}+\bar{\beta}_{i}^{T} \bar{D}_{\bar{\alpha}} \bar{B}_{1}^{T} P_{j} \bar{N}_{1}+\bar{\alpha} \bar{\beta} \bar{B}_{2}^{T} P_{j} \bar{N}_{1}\right]^{T}, \\
\Omega_{4}^{i}=\bar{B}_{1}^{T} P_{j} \bar{B}_{1}+2 \bar{\beta} \bar{B}_{1}^{T} P_{j} \bar{B}_{2}+\bar{\beta} \bar{B}_{2}^{T} P_{j} \bar{B}_{2}+\bar{\beta} \bar{D}^{T} \bar{D}-\gamma^{2} I .
\end{gathered}
$$

By Schur complement, (18) is equivalent to $\bar{\Xi}_{1}^{i}+$ $\sum_{j \in \Re_{U K}^{i}} \rho_{i j} \bar{\Xi}_{2}^{i}<0$, which implies $J_{N}<0$, that is (15), so the proof is completed.

Remark 3. As [18] pointed out, Lyapunov asymptotic stability (LAS) is independent on finite-time stability (FTS); that is, a system which is FTS may not be LAS; conversely a LAS system could not be FTS. In this paper, we investigate more practical fault detection of networked systems in finitetime stochastic stability (FTSS) framework rather than in Lyapunov stochastic stability (LSS) framework [1-9, 13-17]. Especially, if we let $\bar{\sigma}=1$, Theorem 2 will degenerate to fault detection in FTSS framework, in view of this, our conclusion is more general.

Next, sufficient conditions on the existence of modedependent observer-based finite-time fault detection filters would be given, the slack matrix will be constructed with a special structure to eliminate the cross coupling between system matrices and Lyapunov matrices among different operation modes, which allows us to obtain a solution within strict linear matrix inequalities framework for the proposed systems.
Theorem 4. Augmented error system (13) is finite-time stochastically stable with respect to $\left(c_{1}, c_{2}, R, N\right)$ and satisfies $H_{\infty}$ performance level (15) if there exist matrices $\bar{L}_{i}$, $\bar{V}_{i}, X_{i}, Y_{i}, T_{i}, P_{i 1}>0, P_{i 2}, P_{i 3}>0(i \in \Re)$, scalars $\varepsilon_{s}>$ $0(s=1,2,3), \beta \geq 1$, and $\gamma>0$ such that (19) and the following linear matrix inequalities hold:

$$
\left[\begin{array}{ccccccc}
\Xi_{11}^{i} & 0 & \Xi_{12}^{i} & 0 & \sqrt{1-\bar{\alpha}} \Xi_{13}^{i} & \sqrt{\bar{\alpha}} \Xi_{13}^{i} & \Xi_{14}^{i} \\
* & \Xi_{22} & 0 & \Xi_{23}^{i} & \Xi_{24}^{i} & \Xi_{24}^{i}+\bar{\Xi}_{24}^{i} & 0 \\
* & * & \Xi_{33} & 0 & 0 & 0 & \Xi_{34}^{i} \\
* & * & * & \Xi_{44}^{i} & 0 & 0 & 0 \\
* & * & * & * & \Xi_{44}^{i} & 0 & \Xi_{45}^{i} \\
* & * & * & * & * & \Xi_{44}^{i} & \Xi_{45}^{i} \\
* & * & * & * & * & * & \Xi_{55}
\end{array}\right]<0,
$$

$$
\begin{gathered}
\Xi_{11}^{i}=\left[\begin{array}{cc}
-\lambda P_{i 1}-\varepsilon_{1} \delta G^{T} G & -\lambda P_{i 2} \\
* & -\lambda P_{i 3}
\end{array}\right], \\
\Xi_{12}^{i}=\left[\begin{array}{cc}
\sqrt{\bar{\beta}} C^{T} M_{p}^{i} \bar{V}_{i}^{T}-\sqrt{\bar{\beta}} C^{T} \bar{V}_{i}^{T} & \sqrt{1-\bar{\beta}} C^{T} M_{p}^{i} \bar{V}_{i}^{T}-\sqrt{1-\bar{\beta}} C^{T} \bar{V}_{i}^{T} \\
\sqrt{\bar{\beta}} C^{T} \bar{V}_{i}^{T} & \sqrt{1-\bar{\beta}} C^{T} \bar{V}_{i}^{T}
\end{array}\right],
\end{gathered}
$$




$$
\begin{aligned}
& \Xi_{13}^{i}=\left[\begin{array}{cc}
A^{T} X_{i}^{T}+C^{T} \bar{L}_{i}^{T}-C^{T} M_{p}^{i} \bar{L}_{i}^{T} & A^{T} U_{i}^{T}+C^{T} \bar{L}_{i}^{T}-C^{T} M_{p}^{i} \bar{L}_{i}^{T} \\
A^{T} Y_{i}^{T}-C^{T} \bar{L}_{i}^{T} & A^{T} Y_{i}^{T}-C^{T} \bar{L}_{i}^{T}
\end{array}\right], \\
& \Xi_{14}^{i}=\left[\begin{array}{cccc}
-\varepsilon_{2} C^{T} \bar{\Delta}^{T} & 0 & \varepsilon_{3} C^{T} \bar{\Delta}^{T} & 0 \\
0 & 0 & 0 & 0
\end{array}\right], \\
& \Xi_{23}^{i}=\left[\begin{array}{cc}
0 & 0 \\
0 & 0 \\
B_{2}^{T} X_{i}^{T}+B_{2}^{T} Y_{i}^{T} & B_{2}^{T} U_{i}^{T}+B_{2}^{T} Y_{i}^{T}
\end{array}\right] \text {, } \\
& \Xi_{24}^{i}=\left[\begin{array}{cc}
0 & 0 \\
B_{1}^{T} X_{i}^{T}+B_{1}^{T} Y_{i}^{T} & B_{1}^{T} U_{i}^{T}+B_{1}^{T} Y_{i}^{T} \\
\bar{\beta} B_{2}^{T} X_{i}^{T}+\bar{\beta} B_{2}^{T} Y_{i}^{T} & \bar{\beta} B_{2}^{T} U_{i}^{T}+\bar{\beta} B_{2}^{T} Y_{i}^{T}
\end{array}\right], \\
& \bar{\Xi}_{24}^{i}=\left[\begin{array}{cc}
N_{1}^{T} X_{i}^{T}+N_{1}^{T} Y_{i}^{T} & N_{1}^{T} U_{i}^{T}+N_{1}^{T} Y_{i}^{T} \\
0 & 0 \\
0 & 0
\end{array}\right] \text {, } \\
& \Xi_{34}^{i}=\left[\begin{array}{llll}
0 & 0 & 0 & \bar{V}_{i} M_{p}^{i} \\
0 & 0 & 0 & \bar{V}_{i} M_{p}^{i}
\end{array}\right], \quad \Xi_{45}^{i}=\left[\begin{array}{cccc}
0 & \bar{L}_{i} M_{p}^{i} & 0 & 0 \\
0 & \bar{L}_{i} M_{p}^{i} & 0 & 0
\end{array}\right], \\
& \Xi_{44}^{i}=\left[\begin{array}{cc}
\Upsilon_{j 1}-X_{i}-X_{i}^{T} & \Upsilon_{j 2}-Y_{i}-U_{i}^{T} \\
* & \Upsilon_{j 3}-Y_{i}-Y_{i}^{T}
\end{array}\right], \\
& \Xi_{22}^{i}=\operatorname{diag}\left\{-\varepsilon_{1} I,-\gamma^{2} I,-\gamma^{2} I\right\}, \\
& \Xi_{55}^{i}=\operatorname{diag}\left\{-\varepsilon_{2} I,-\varepsilon_{2} I,-\varepsilon_{3} I,-\varepsilon_{3} I\right\}, \quad \Xi_{33}^{i}=\operatorname{diag}\{-I,-I\} .
\end{aligned}
$$

Then, there exists mode-dependent observer-based finite-time fault detection filters such that the augmented error systems (13) with partly unknown transition probabilities are finite-time stochastically stable with $H_{\infty}$ performance level (15). Moreover, if LMIs (34) have feasible solution, the desired finite-time fault detection filters can be given by

$$
L_{i}=Y_{i}^{-1} \bar{L}_{i}, \quad V_{i}=\bar{V}_{i} \quad(i \in \Re) .
$$

Proof. For an arbitrary matrix $R_{i}=\left[\begin{array}{l}X_{i} Y_{i} \\ U_{i} Y_{i}\end{array}\right], \forall i \in \mathfrak{R}$, assuming that $Y_{i}$ is inverse, we have the following fact:

$$
\begin{gathered}
\left(\frac{1}{\rho_{K}^{i}} P_{K}^{i}-R_{i}\right)\left(\frac{1}{\rho_{K}^{i}} P_{K}^{i}\right)^{-1}\left(\frac{1}{\rho_{K}^{i}} P_{K}^{i}-R_{i}\right)^{T} \geq 0 ; \\
\left(P_{j}-R_{i}\right) P_{j}^{-1}\left(P_{j}-R_{i}\right)^{T} \geq 0 .
\end{gathered}
$$

where $\bar{\Xi}_{14}=\operatorname{diag}\left\{R_{i}^{T}, R_{i}^{T}, R_{i}^{T}\right\}$ and $\bar{\Xi}_{22}=\operatorname{diag}\left\{\Upsilon_{j}-R_{i}^{T}-R_{i}, \Upsilon_{j}-\right.$ $\left.R_{i}^{T}-R_{i}, \Upsilon_{j}-R_{i}^{T}-R_{i}\right\}$.

Define matrices variables $\bar{L}_{i}=Y_{i} L_{i}$ and $\bar{V}_{i}=V_{i}$, noting that a congruence transformation to (18) by $J_{1}=\operatorname{diag}\{I, I, I$, $\left.I, I, \Upsilon_{j}^{-1} R_{i}^{T}, \Upsilon_{j}^{-1} R_{i}^{T}, \Upsilon_{j}^{-1} R_{i}^{T}\right\}$, we obtain

$$
\left[\begin{array}{ccc}
\Xi_{11} & \Xi_{12}^{T} & \Xi_{13}^{T} \bar{\Xi}_{14} \\
* & \operatorname{diag}\{I, I\} & 0 \\
* & * & \bar{\Xi}_{22}
\end{array}\right]<0,
$$

Then, we have $\Upsilon_{j}-R_{i}^{T}-R_{i} \geq-R_{i} \Upsilon_{j}^{-1} R_{i}^{T}$; performing

$$
\begin{aligned}
& \bar{A}_{i}^{T} R_{i}^{T}= {\left[\begin{array}{cc}
A^{T} X_{i}^{T}+C^{T} \bar{L}_{i}^{T}-C^{T} M_{p}^{i} \bar{L}_{i}^{T} & A^{T} U_{i}^{T}+C^{T} \bar{L}_{i}^{T}-C^{T} M_{p}^{i} \bar{L}_{i}^{T} \\
A^{T} X_{i}^{T}-C^{T} \bar{L}_{i}^{T} & A^{T} X_{i}^{T}-C^{T} \bar{L}_{i}^{T}
\end{array}\right] } \\
&+\left[\begin{array}{cc}
-C^{T} \Delta^{T}(k) M_{p}^{i} \bar{L}_{i}^{T} & -C^{T} \Delta^{T}(k) M_{p}^{i} \bar{L}_{i}^{T} \\
0 & 0
\end{array}\right]=\underline{A}_{i}^{T}+\widehat{A}^{T} F^{T}(k) \breve{A}_{i}^{T} \\
& \bar{C}_{i}^{T}=\left[\begin{array}{c}
C^{T} M_{p}^{i} \bar{V}_{i}^{T}-C^{T} \bar{V}_{i}^{T} \\
C^{T} \bar{V}_{i}^{T}
\end{array}\right]+\left[\begin{array}{c}
C^{T} \Delta^{T}(k) M_{p}^{i} \bar{V}_{i}^{T} \\
0
\end{array}\right]=\underline{C}_{i}^{T}+\widehat{C}^{T} F^{T}(k) \breve{C}_{i}^{T},
\end{aligned}
$$


where

$$
\begin{aligned}
& \underline{A}_{i}^{T}=\left[\begin{array}{cc}
A^{T} X_{i}^{T}+C^{T} \bar{L}_{i}^{T}-C^{T} M_{p}^{i} \bar{L}_{i}^{T} & A^{T} U_{i}^{T}+C^{T} \bar{L}_{i}^{T}-C^{T} M_{p}^{i} \bar{L}_{i}^{T} \\
A^{T} X_{i}^{T}-C^{T} \bar{L}_{i}^{T} & A^{T} X_{i}^{T}-C^{T} \bar{L}_{i}^{T}
\end{array}\right], \\
& \widehat{A}^{T}=\left[\begin{array}{c}
-C^{T} \bar{\Delta}^{T} \\
0
\end{array}\right], \quad \breve{A}_{i}^{T}=\left[\begin{array}{ll}
M_{p}^{i} \bar{L}_{i}^{T} & M_{p}^{i} \bar{L}_{i}^{T}
\end{array}\right], \quad \underline{C}_{i}^{T}=\left[\begin{array}{c}
C^{T} M_{p}^{i} \bar{V}_{i}^{T}-C^{T} \bar{V}_{i}^{T} \\
C^{T} \bar{V}_{i}^{T}
\end{array}\right], \\
& \widehat{C}^{T}=C^{T} \bar{\Delta}^{T}, \quad \breve{C}_{i}^{T}=\left[\begin{array}{c}
M_{p}^{i} \bar{V}_{i}^{T} \\
0
\end{array}\right] .
\end{aligned}
$$

Then (38) can be rewritten as

where

$$
\begin{aligned}
\Sigma+ & \Sigma_{1} F(k) \Sigma_{2}+\Sigma_{2}^{T} F^{T}(k) \Sigma_{1}^{T}+\Sigma_{3} F(k) \Sigma_{4} \\
& +\Sigma_{4}^{T} F^{T}(k) \Sigma_{3}^{T}<0
\end{aligned}
$$

$$
\begin{aligned}
& \Sigma=\left[\begin{array}{ccc}
\Xi_{11} & \bar{\Xi}_{12}^{T} & \bar{\Xi}_{13}^{T} \bar{\Xi}_{14} \\
* & \operatorname{diag}\{I, I\} & 0 \\
* & * & \bar{\Xi}_{22}
\end{array}\right] \\
& \bar{\Xi}_{13}^{T}=\left[\begin{array}{ccc}
0 & \sqrt{1-\bar{\alpha}} \underline{A}_{i}^{T} & \sqrt{\bar{\alpha}} \underline{\underline{A}}_{i}^{T} \\
0 & 0 & \sqrt{\bar{\alpha}} \bar{N}_{1}^{T} \\
\sqrt{\bar{\beta}(1-\bar{\beta})} \bar{B}_{2}^{T} & \sqrt{1-\bar{\alpha}}\left(\bar{B}_{2}^{T}+\bar{\beta}_{\bar{B}}^{T}\right) & \sqrt{\bar{\alpha}}\left(\bar{B}_{2}^{T}+\bar{\beta} \bar{B}_{2}^{T}\right)
\end{array}\right], \\
& \bar{\Xi}_{12}^{T}=\left[\begin{array}{cc}
\sqrt{\bar{\beta}} \underline{C}_{i}^{T} & \sqrt{1-\bar{\beta}} \underline{C}_{i}^{T} \\
0 & 0 \\
\sqrt{\beta} \bar{D}^{T} & 0
\end{array}\right], \quad \Sigma_{1}^{T}=[\underbrace{\widehat{A}, \underbrace{0, \ldots, 0}_{7}}_{7}]^{T}, \quad \Sigma_{2}^{T}=[\underbrace{0, \ldots, 0}_{6}, \breve{A}_{i}^{T}, \breve{A}_{i}^{T}], \\
& \Sigma_{3}^{T}=[\widehat{C}, \underbrace{0, \ldots, 0}_{7}]^{T}, \quad \Sigma_{4}^{T}=\left[0,0,0, \breve{C}_{i}^{T}, \breve{C}_{i}^{T}, 0,0,0\right] .
\end{aligned}
$$

From Schur Complement, for any scalars $\varepsilon_{i}>0(i=2,3)$, we get

$$
\left[\begin{array}{ccccc}
\Sigma & \varepsilon_{2} \Sigma_{1}^{T} & \Sigma_{2} & \varepsilon_{3} \Sigma_{3}^{T} & \Sigma_{4} \\
* & -\varepsilon_{2} I & 0 & 0 & 0 \\
* & * & -\varepsilon_{2} I & 0 & 0 \\
* & * & * & -\varepsilon_{3} I & 0 \\
* & * & * & * & -\varepsilon_{3} I
\end{array}\right]<0
$$

Now, partition $P_{i}$ as $P_{i}=\left[\begin{array}{cc}P_{i 1} & P_{i 2} \\ * & P_{i 3}\end{array}\right]$; then define matrics $\Upsilon_{j} \triangleq \sum_{j \in \Re_{K}^{i}} \sigma_{i j} P_{j}=\sum_{j \in \Re_{K}^{i}} \sigma_{i j}\left[\begin{array}{cc}P_{j 1} & P_{j 2} \\ * & P_{j 3}\end{array}\right] \triangleq\left[\begin{array}{cc}\Upsilon_{j 1} & \Upsilon_{j 2} \\ * & \Upsilon_{j 3}\end{array}\right]$. If we replace $\bar{L}_{i}=Y_{i} L_{i}$ and $\bar{V}_{i}=V_{i}$ into (43), we readily obtain (34). From Theorem 2, augmented error systems (13) will be finite-time stochastically stable with $H_{\infty}$ performance level (15). Meanwhile, if the solution of (34) exists, the admissible finite-time fault detection filters are given by (36). The proof is completed.

From a viewpoint of computation, it should be noted that the conditions in Theorem 4 are still not standard linear matrix inequalities (LMIs) conditions due to (18). Actually, conditions (18) can also be guaranteed by LMIs conditions once the values of $\bar{\sigma}$ is set. For given positive scalar $\lambda$, it is easy to check that condition (18) is guaranteed by imposing condition $\vartheta \leq \underline{\lambda}=\min _{i \in \mathfrak{R}} \lambda_{\text {min }}\left(\widetilde{P}_{i}\right)<1$ and

$$
\begin{gathered}
\vartheta R \leq P_{i} \leq R, \\
\frac{c_{1}^{2}}{\vartheta}<\frac{c_{2}^{2}}{\bar{\sigma}^{N}} .
\end{gathered}
$$


Then, inequality (18) can be converted into the following LMI by using Schur Complement:

$$
\left[\begin{array}{cc}
-\frac{c_{2}^{2}}{\bar{\sigma}^{N}} & c_{1} \\
* & -\vartheta
\end{array}\right]<0 .
$$

Thus, once $\bar{\sigma}$ is fixed, the feasibility of (18) in Theorem 4 can be translated into LMI-based conditions (44) and (46). Theorem 4 can be solved by Matlab's LMI toolbox [21].

Remark 5. As the special cases of partly unknown transition probabilities, when all the transition probabilities are completely accessible $\left(\mathfrak{R}_{K}^{i}=\mathfrak{R}, \mathfrak{R}_{U K}^{i}=\Phi\right)$ and completely inaccessible $\left(\mathfrak{R}_{U K}^{i}=\mathfrak{R}, \mathfrak{R}_{K}^{i}=\Phi\right)$, the underlying systems are the traditional Markovian jump systems and the switched systems under arbitrary switching, respectively. Correspondingly, the fault detection results can be found in some existing references, [10] investigated linear discretetime Markovian jump systems (completely accessible), [11] studied linear discrete-time switched systems (completely inaccessible), [22] considered transition probabilities with polytopic uncertainties which require the knowledge of uncertainties structure and it can still be viewed as accessible. Therefore, finite-time fault detection with partly unknown transition probabilities is a more natural assumption to the Markovian jump systems and hence covers the existing ones. Furthermore, when $\bar{\alpha}=1$ and $\bar{\beta}=1$, then augmented error systems (13) with RON and ROF are the usually nonlinear system [19] and fault detection system [10-17]; from this view, (13) is also a more comprehensive networked systems model.

\section{Numerical Example and Simulation}

Consider the nonlinear networked systems (1) with the following parameters:

$$
\begin{array}{ll}
A=\left[\begin{array}{cc}
-0.2 & 0.1 \\
-0.2 & 0.3
\end{array}\right], & B_{1}=\left[\begin{array}{l}
0.7 \\
0.2
\end{array}\right], \quad B_{2}=\left[\begin{array}{c}
0 \\
-0.3
\end{array}\right], \\
C=\left[\begin{array}{cc}
0.2 & 0.5 \\
0.1 & -0.3
\end{array}\right], & N_{1}=\left[\begin{array}{cc}
0 & 0.3 \\
0.1 & -0.5
\end{array}\right], \\
G=\left[\begin{array}{cc}
-0.1 & 0.3 \\
0 & 0.2
\end{array}\right], & R=\left[\begin{array}{ll}
1 & 0 \\
0 & 1
\end{array}\right], \quad N=20 .
\end{array}
$$

Attention is focused on the design of mode-dependent observer-based finite-time fault detection filters, which make the augmented error systems (13) finite-time stochastically stable with $H_{\infty}$ performance level (15). If we consider two channels data transmission networked systems, according to the transmission pattern presented in Section 2, the transmission matrices are constructed as

$$
\begin{array}{ll}
M_{2}^{2,1} \triangleq \operatorname{diag}\{1,1\}, & M_{2}^{0,1} \triangleq \operatorname{diag}\{0,0\}, \\
M_{2}^{1,1} \triangleq \operatorname{diag}\{1,0\}, & M_{2}^{1,2} \triangleq \operatorname{diag}\{0,1\} .
\end{array}
$$

With above data transmission pattern, from Remark 5, we consider the Case II with partly unknown transition probabilities in Table 1, where "?" means that element is unknown. As the special cases of Case II, corresponding results of traditional Markovian jump systems (Case I) and switched systems (Case III) can be included in our theorems. By Theorem 4, for the given $c_{1}=0.6, \bar{\alpha}=0.5$, and $\delta=0.4$, the suboptimal finite-time fault detection performance level $\gamma^{*}$ is obtained in Table 2 . From Table 2, it can be easily seen that finite-time fault detection performance level $\gamma^{*}$ is dependent on ROFs probability $\bar{\beta}$ and RONs probability $\bar{\alpha}$, finite-time stability index $\bar{\sigma}$, quantization level $\chi_{i}$, and the information of transition probability matrices, which show the effectiveness of our discussion.

Assuming that the parameters are given by $u_{0}^{1}=u_{0}^{2}=$ $1, \chi_{1}=\chi_{2}=0.8, \bar{\beta}=0.6, \bar{\sigma}=1.3, c_{1}=0.6, \bar{\alpha}=0.5, \delta=$ 0.4 , and $\gamma=1.1$, by applying (34), (36), (44), and (46) of Theorem 4, mode-dependent observer-based finite-time fault detection filter matrices $L_{i}^{j}$ and $V_{i}^{j}(i=1,2,3,4 ; j=1,2,3)$ can be obtained as follows:

$$
\begin{array}{ll}
L_{1}^{1}=\left[\begin{array}{cc}
-0.0458 & -0.0346 \\
0.0889 & -0.1042
\end{array}\right], & V_{1}^{1}=\left[\begin{array}{ll}
-0.0167 & 0.0385
\end{array}\right], \\
L_{2}^{1}=\left[\begin{array}{cc}
-1.0599 & -5.6530 \\
1.7666 & 4.2394
\end{array}\right], \quad V_{2}^{1}=\left[\begin{array}{ll}
-0.0431 & -0.2377
\end{array}\right], \\
L_{3}^{1}=\left[\begin{array}{cc}
-0.0714 & -0.2247 \\
0.0941 & -0.4006
\end{array}\right], \quad V_{3}^{1}=\left[\begin{array}{ll}
-0.0369 & -0.1734
\end{array}\right], \\
L_{4}^{1}=\left[\begin{array}{cc}
-0.0078 & -0.0235 \\
0.1124 & -0.1258
\end{array}\right], & V_{4}^{1}=\left[\begin{array}{ll}
0.0479 & 0.0529
\end{array}\right], \\
L_{1}^{2}=\left[\begin{array}{cc}
-0.0658 & -0.0699 \\
0.1156 & -0.1768
\end{array}\right], & V_{1}^{2}=\left[\begin{array}{ll}
-0.0155 & 0.0426
\end{array}\right], \\
L_{2}^{2}=\left[\begin{array}{cc}
-0.0761 & -1.3612 \\
0.9768 & 1.9094
\end{array}\right], & V_{2}^{2}=\left[\begin{array}{ll}
-0.0260 & -0.1211
\end{array}\right], \\
L_{3}^{2}=\left[\begin{array}{cc}
-0.2241 & -0.6533 \\
0.1698 & -0.7305
\end{array}\right], & V_{3}^{2}=\left[\begin{array}{ll}
-0.0321 & -0.1185
\end{array}\right], \\
L_{4}^{2}=\left[\begin{array}{cc}
0.0272 & -0.0135 \\
0.1279 & -0.2607
\end{array}\right], & V_{4}^{2}=\left[\begin{array}{ll}
0.0679 & 0.0757
\end{array}\right], \\
L_{1}^{3}=\left[\begin{array}{cc}
-0.0317 & -0.0356 \\
0.0835 & -0.0987
\end{array}\right], & V_{1}^{3}=\left[\begin{array}{ll}
-0.0098 & 0.0267
\end{array}\right], \\
L_{2}^{3}=\left[\begin{array}{cc}
0.2694 & 5.7487 \\
-1.1041 & -3.3997
\end{array}\right], & V_{2}^{3}=\left[\begin{array}{ll}
-0.0443 & -0.2361
\end{array}\right], \\
L_{3}^{3}=\left[\begin{array}{cc}
-0.0561 & -0.2962 \\
0.0792 & -0.2882
\end{array}\right], & V_{3}^{3}=\left[\begin{array}{ll}
-0.0375 & -0.1713
\end{array}\right], \\
L_{4}^{3}=\left[\begin{array}{cc}
-0.0143 & -0.0284 \\
0.1083 & -0.1125
\end{array}\right], & V_{4}^{3}=\left[\begin{array}{ll}
0.0541 & 0.0435
\end{array}\right],
\end{array}
$$

To demonstrate the effectiveness of designed finite-time fault detection filter, for $k=0,1, \ldots, 20$, unknown disturbance input $d(k)$ is assumed to be band-limited white noise 


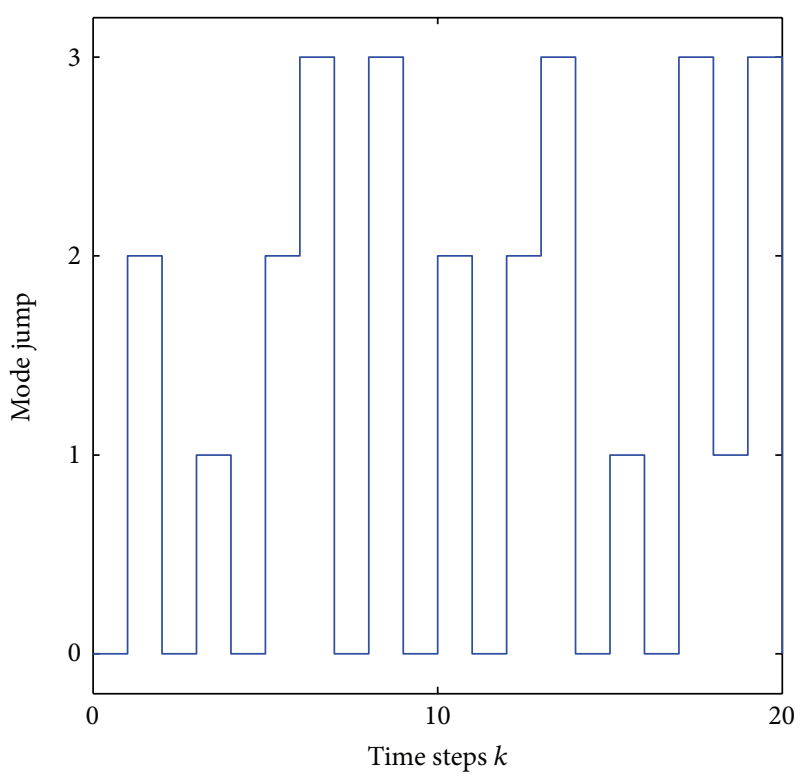

(a)

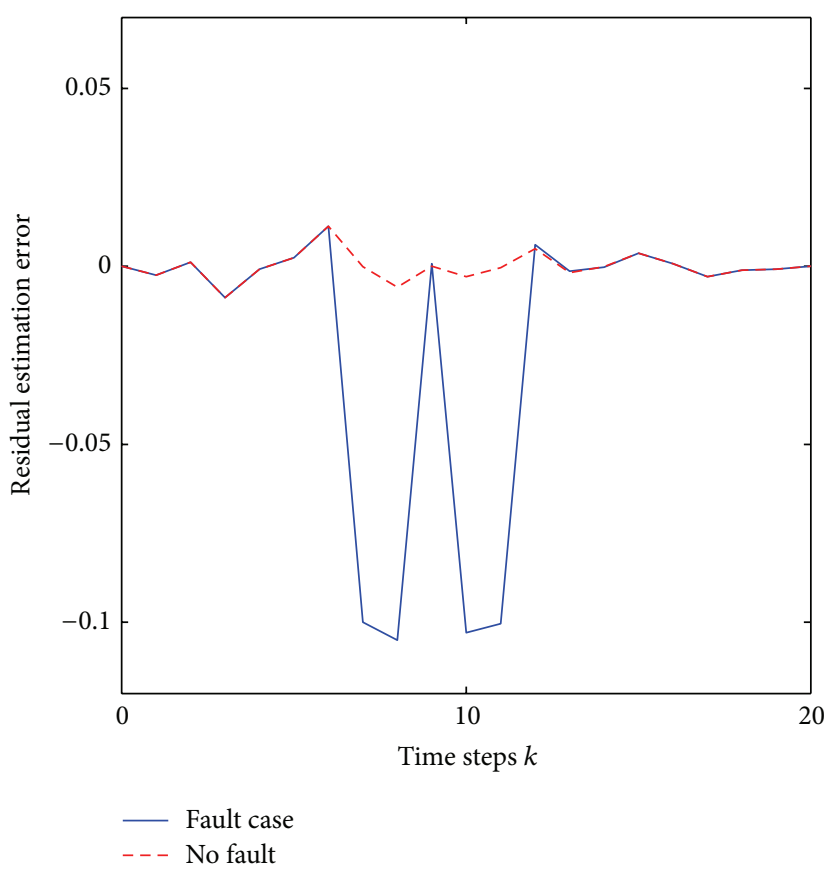

(c)

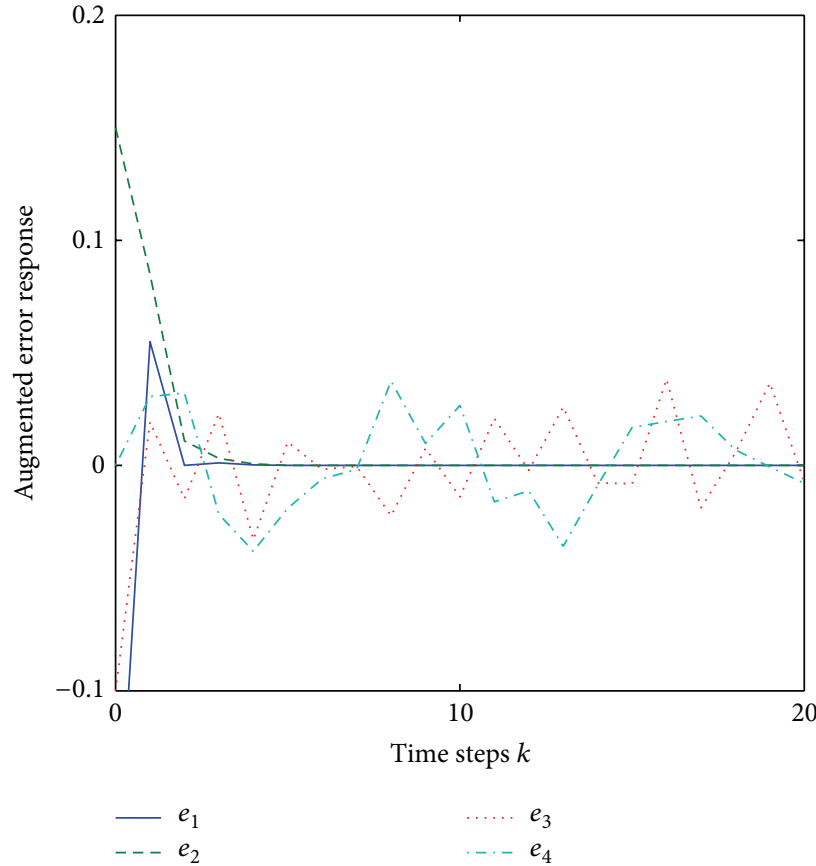

(b)

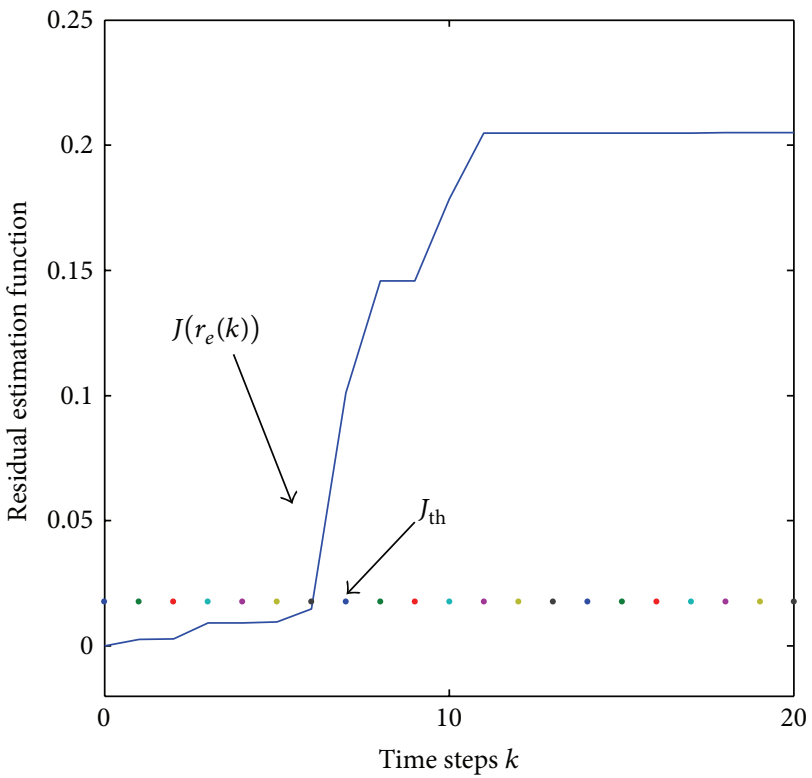

(d)

FIGURE 1: Corresponding simulation of Case I.

with power of 0.05 , and the fault signal $f(k)$ is simulated as a square wave of 0.1 amplitude that occurred from 8 to 14 steps and the nonlinear function is given by $g(x(k))=$ $\sin (0.1 \times x(k))$. Under Cases I, II, and III, the initial state of augmented error systems (13) is assumed as $e(0)=$ $\left[\begin{array}{llll}-0.2 & 0.15 & -0.1 & 0\end{array}\right]^{T}$, corresponding evolution of residual estimation error signal $r_{e}(k)$ and residual evaluation function $J\left(r_{e}\right)$ are shown in Figures 1, 2, and 3, respectively. For given $k_{0}=0$ and $\kappa=20$, the threshold $J_{\text {th }}$ can be determined by utilizing 300 Monte Carlo simulations in Table 3; from Table 3, we observe that, when $k=8, J\left(r_{e}\right) \geq J_{\text {th }}$, for the first time, which means that the fault $f(k)$ can be detected 

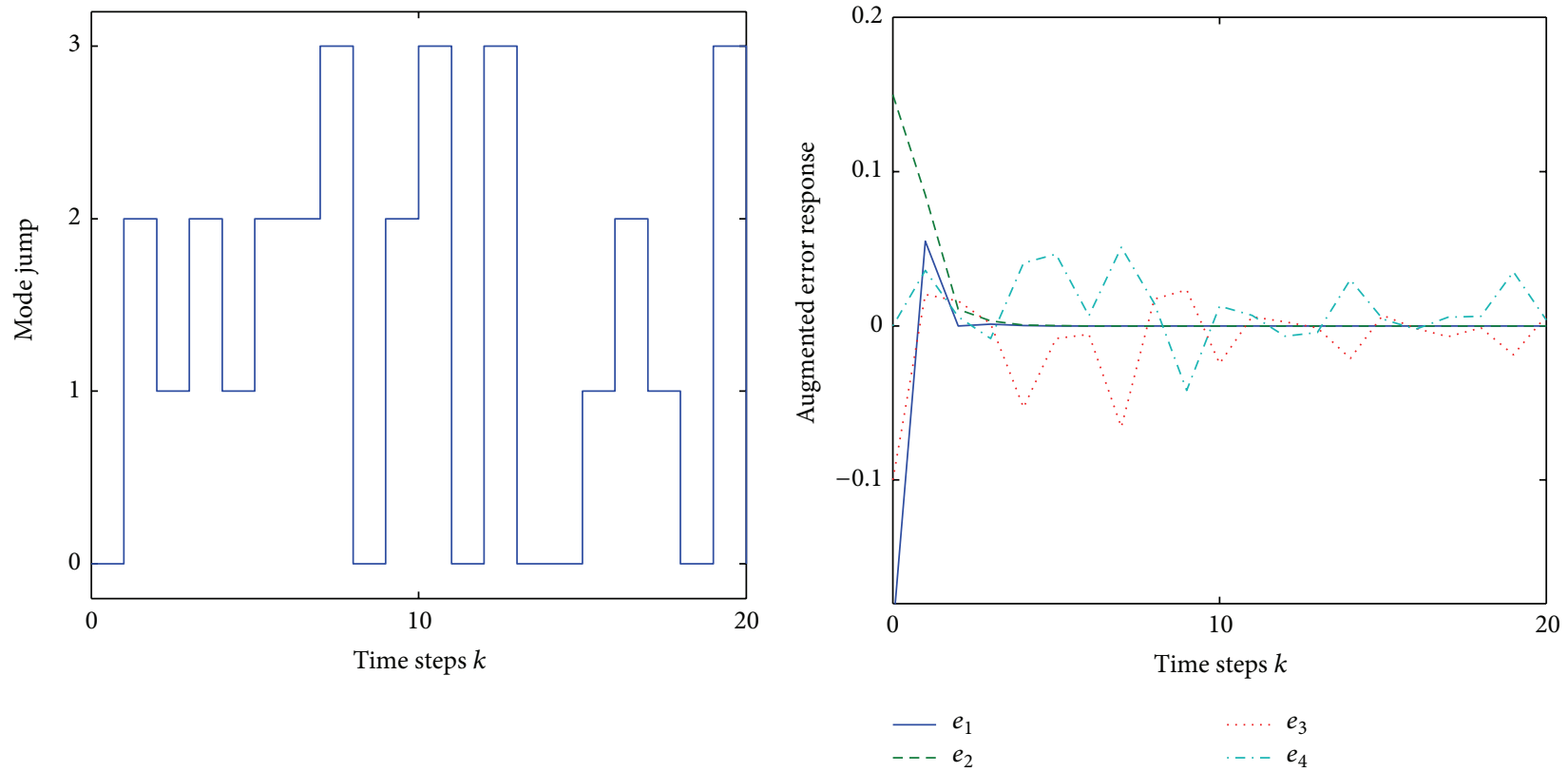

(a)

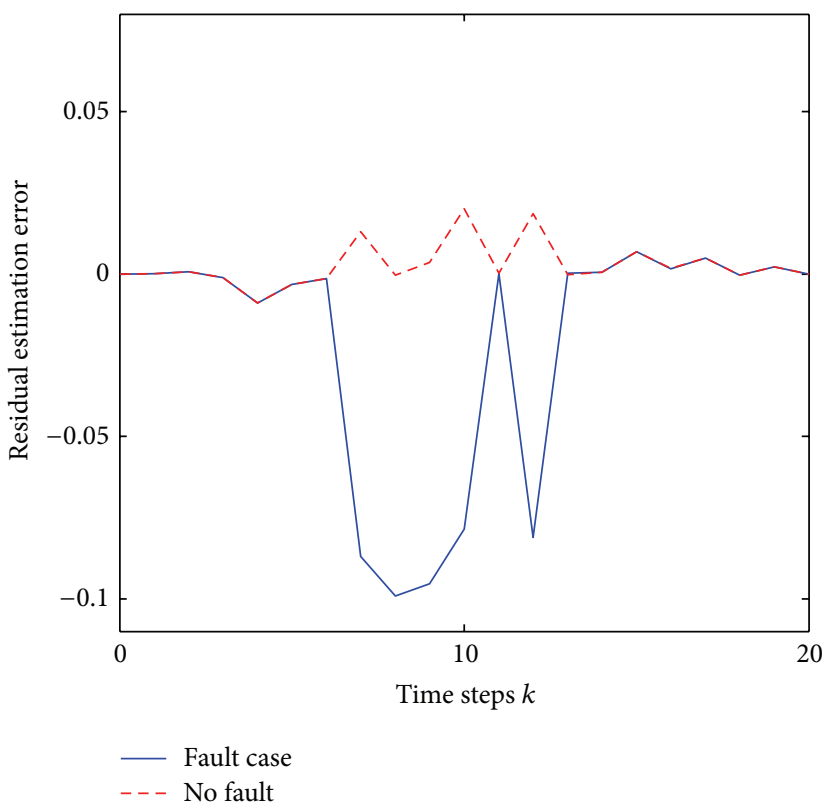

(b)

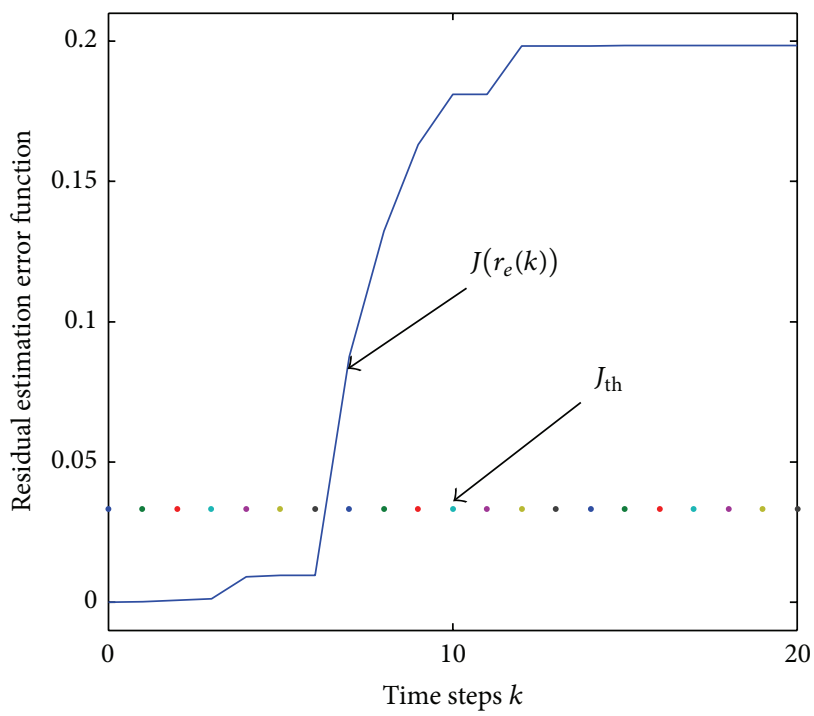

(c)

(d)

FIGURE 2: Corresponding simulation of Case II.

TABLE 1: Different transition probabilities matrices cases.

\begin{tabular}{|c|c|c|c|}
\hline $\begin{array}{l}\text { Completely known } \\
\text { (Case I) }\end{array}$ & $\begin{array}{l}\text { Partly unknow } \\
\text { (Case II) }\end{array}$ & & $\begin{array}{c}\text { Completely unknown } \\
\text { (Case III) }\end{array}$ \\
\hline$\left[\begin{array}{llll}0.1 & 0.2 & 0.3 & 0.4\end{array}\right]$ & $\begin{array}{llll}0.1 & 0.2 & 0.3 & 0\end{array}$ & 0.47 & {$[? ? ? ? ?]$} \\
\hline $0.1 \quad 0.6 \quad 0.1 \quad 0.2$ & $\begin{array}{llll}? & 0.6 & ? & 0\end{array}$ & 0.2 & ? ? ? ? \\
\hline $\begin{array}{llll}0.5 & 0.1 & 0.2 & 0.2\end{array}$ & $0.5 ? ?$ & $?$ & ? ? ? ? \\
\hline$\left[\begin{array}{llll}0.6 & 0.1 & 0.2 & 0.1\end{array}\right]$ & $0.1 \quad 0.2 \quad 0$ & $0.1]$ & {$\left[\begin{array}{lllll}\text { ? } & ? & ? & ?\end{array}\right]$} \\
\hline
\end{tabular}

as soon as its occurrence, respectively, so the effectiveness of proposed finite time fault detection problem is illustrated.

\section{Conclusion}

This paper is concerned with the problem of finite-time fault detection for large-scale networked systems. A Makovian jump systems model with partly unknown transition 


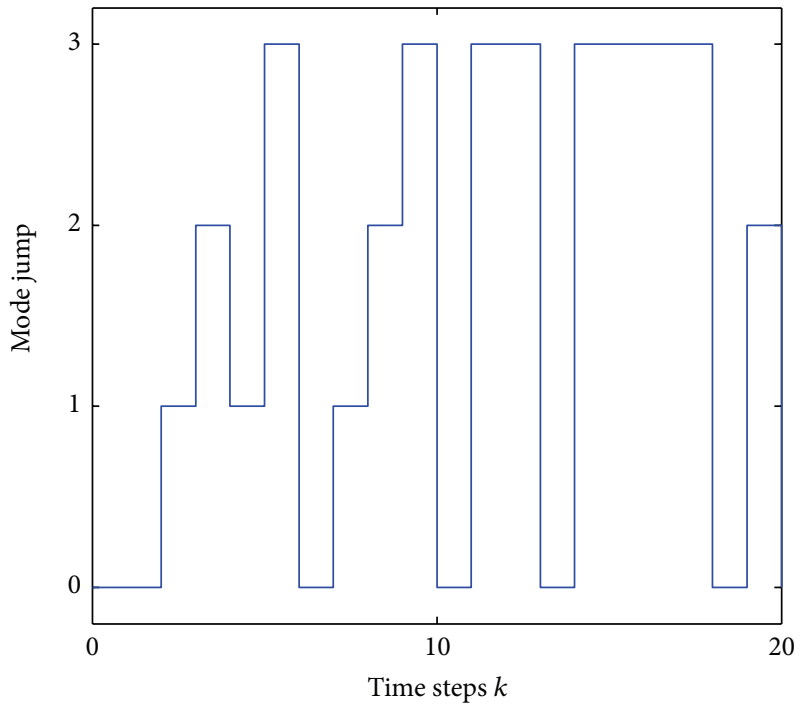

(a)

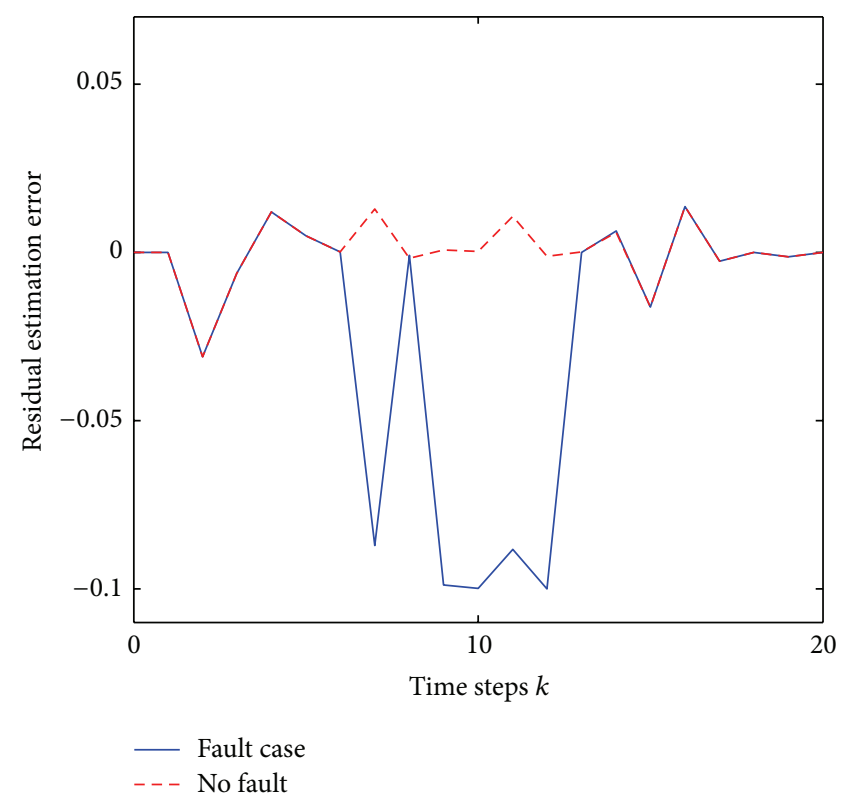

(c)

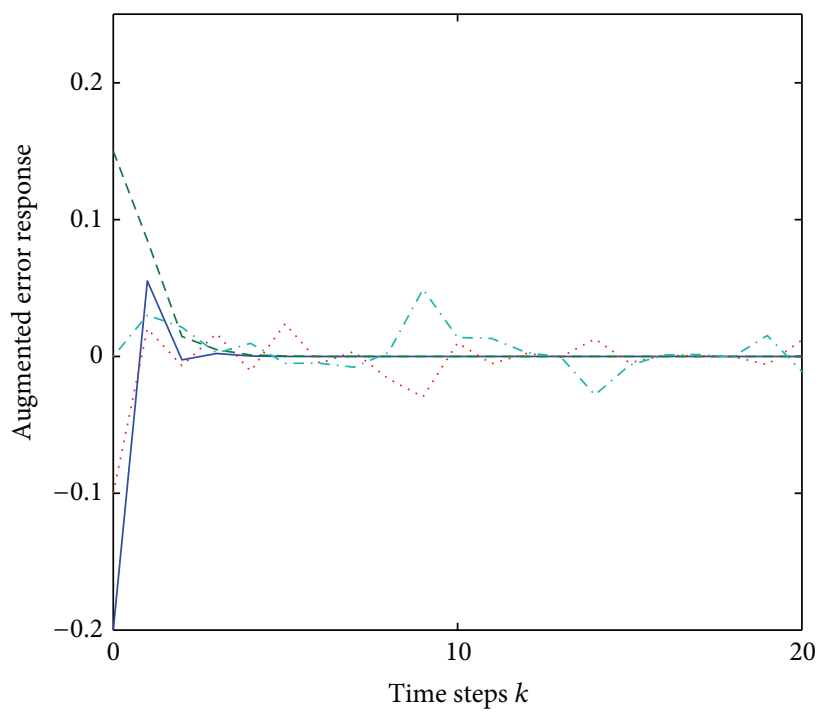

$-e_{1}$

...... $e_{3}$

(b)

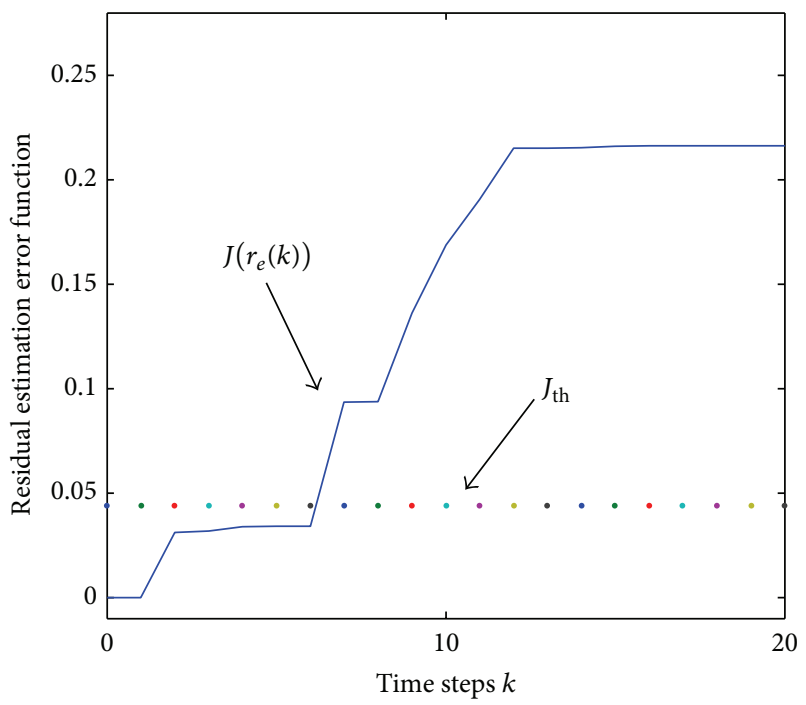

(d)

FIGURE 3: Corresponding simulation of Case III.

TABLE 2: Corresponding minimum finite-time $H_{\infty}$ attenuation level $\gamma^{*}$ for different cases.

\begin{tabular}{|c|c|c|c|c|}
\hline & $\begin{array}{c}\bar{\sigma}=1.1, \bar{\beta}=0.4 \\
\chi_{1}=\chi_{2}=0.3\end{array}$ & $\begin{array}{c}\bar{\sigma}=1.5, \bar{\beta}=0.4 \\
\chi_{1}=\chi_{2}=0.3\end{array}$ & $\begin{array}{c}\bar{\sigma}=1.5, \bar{\beta}=0.7 \\
\chi_{1}=\chi_{2}=0.3\end{array}$ & $\begin{array}{c}\bar{\sigma}=1.5, \bar{\beta}=0.7 \\
\chi_{1}=\chi_{2}=0.8\end{array}$ \\
\hline Completely known & 1.0001 & 1.0022 & 1.0102 & 1.0103 \\
\hline Partly unknown & 1.0001 & 1.0083 & 1.0146 & 1.0145 \\
\hline Completely unknown & 1.0001 & 1.0113 & 1.0895 & 1.0895 \\
\hline
\end{tabular}


TABLE 3: Corresponding threshold and residual evolution function value for different cases.

\begin{tabular}{lccr}
\hline & Completely known & Partly unknown & Completely unknown \\
\hline Threshold & $J_{\text {th }}=0.0177$ & $J_{\text {th }}=0.0333$ & $J_{\text {th }}=0.0442$ \\
\hline Residual evolution & $0.0148=J(7)<$ & $0.0096=J(7)<$ & $0.0343=J(7)<$ \\
Error function & $J_{\text {th }}<J(8)=0.1012$ & $J_{\text {th }}<J(8)=0.0875$ & $J_{\text {th }}<J(8)=0.0937$ \\
\hline
\end{tabular}

probabilities is introduced to describe multiple channels data transmission pattern, while the cases with completely known or completely unknown transition probabilities have been investigated as its special cases. The randomly occurring nonlinearities and randomly occurring faults are also introduced to reflect the limited capacity of the communication network resulting from the noisy environment and probabilistic communication failures. Based on this, more natural model, finite-time fault detection of nonlinear large-scale networked systems, is formulated as nonlinear finite-time $H_{\infty}$ attention problem. The main objective is to design mode-dependent observer-based finite-time fault detection filter such that the error between residual signal and fault signal is made as small as possible. Simulations are given to illustrate the effectiveness of proposed design techniques.

\section{Conflict of Interests}

The authors declare that there is no conflict of interests regarding the publication of this paper.

\section{Acknowledgments}

The authors would like to thank the editor and the reviewers for their helpful suggestions to improve the quality of this correspondence. This work is supported by e National Natural Science Foundation of China by Grant nos. 61104027, 61174107, and 61034006.

\section{References}

[1] W. Zhang, M. S. Branicky, and S. M. Phillips, "Stability of networked control systems," IEEE Control Systems Magazine, vol. 21, no. 1, pp. 84-99, 2001.

[2] J. P. Hespanha, P. Naghshtabrizi, and Y. G. Xu, "A survey of recent results in networked control systems," Proceedings of the IEEE, vol. 95, no. 1, pp. 138-162, 2007.

[3] G.-P. Liu, Y. Q. Xia, R. D. Rees, and W. S. Hu, "Design and stability criteria of networked predictive control systems with random network delay in the feedback channel," IEEE Transactions on Systems, Man and Cybernetics C: Applications and Reviews, vol. 37, no. 2, pp. 173-184, 2007.

[4] D. Yue, E. G. Tian, Z. D. Wang, and J. Lam, "Stabilization of systems with probabilistic interval input delays and its applications to networked control systems," IEEE Transactions on Systems, Man, and Cybernetics A: Systems and Humans, vol. 39, no. 4, pp. 939-945, 2009.

[5] D. Ye, Y. Long, and G.-H. Yang, "Finite-frequency filter design for networked control systems with missing measurements," Mathematical Problems in Engineering, vol. 2013, Article ID 825143, 8 pages, 2013.
[6] L. Zhang and D. Hristu-Varsakelis, "Communication and control co-design for networked control systems," Automatica, vol. 42, no. 6, pp. 953-958, 2006.

[7] D.-S. Kim, D.-H. Choi, and P. Mohapatra, "Real-time scheduling method for networked discrete control systems," Control Engineering Practice, vol. 17, no. 5, pp. 564-570, 2009.

[8] J. N. Li, L. F. Wei, C. Liu, and H. Y. Yu, " $H_{\infty}$ control of network-based systems with packet disordering and packet loss compensation," Mathematical Problems in Engineering, vol. 2013, Article ID 836314, 9 pages, 2013.

[9] S. Hu and W.-Y. Yan, "Stability of networked control systems under a multiple-packet transmission policy," IEEE Transactions on Automatic Control, vol. 53, no. 7, pp. 1706-1711, 2008.

[10] M. Y. Zhong, S. X. Ding, J. Lam, and H. Wang, "Fault detection for Markovian jump systems," IEE Proceedings-Control Theory and Application, vol. 152, no. 4, pp. 397-402, 2005.

[11] D. Wang, W. Wang, and P. Shi, "Robust fault detection for switched linear systems with state delays," IEEE Transactions on Systems, Man, and Cybernetics B: Cybernetics, vol. 39, no. 3, pp. 800-805, 2009.

[12] Y. Yin, P. Shi, and F. Liu, "Gain-scheduled robust fault detection on time-delay stochastic nonlinear systems," IEEE Transactions on Industrial Electronics, vol. 58, no. 10, pp. 4908-4916, 2011.

[13] H. J. Fang, H. Ye, and M. Y. Zhong, "Fault diagnosis of networked control systems," Annual Reviews in Control, vol. 31, no. 1, pp. 55-68, 2007.

[14] H. L. Dong, Z. D. Wang, J. Lam, and H. J. Gao, "Fuzzy-modelbased robust fault detection with stochastic mixed time delays and successive packet dropouts," IEEE Transactions on Systems, Man, and Cybernetics B: Cybernetics, vol. 42, no. 2, pp. 365-376, 2012.

[15] Z. Mao, B. Jiang, and P. Shi, "Protocol and fault detection design for nonlinear networked control systems," IEEE Transactions on Circuits and Systems II: Express Briefs, vol. 56, no. 3, pp. 255-259, 2009.

[16] X. He, Z. Wang, and D. H. Zhou, "Robust fault detection for networked systems with communication delay and data missing," Automatica, vol. 45, no. 11, pp. 2634-2639, 2009.

[17] Y. Zhang and H. J. Fang, "Fault detection for nonlinear networked systems with random packet dropout and probabilistic interval delay," International Journal of Adaptive Control and Signal Processing, vol. 25, no. 12, pp. 1074-1086, 2011.

[18] F. Amato, M. Ariola, and P. Dorato, "Finite-time control of linear systems subject to parametric uncertainties and disturbances," Automatica, vol. 37, no. 9, pp. 1459-1463, 2001.

[19] Z. D. Wang, Y. Wang, and Y. Liu, "Global synchronization for discrete-time stochastic complex networks with randomly occurred nonlinearities and mixed time delays," IEEE Transactions on Neural Networks, vol. 21, no. 1, pp. 11-25, 2010. 
[20] M. Fu and L. Xie, "The sector bound approach to quantized feedback control," IEEE Transactions on Automatic Control, vol. 50, no. 11, pp. 1698-1711, 2005.

[21] S. Boyd, L. el Ghaoui, E. Feron, and V. Balakrishnan, Linear Matrix Inequalities in System and Control Theory, SIAM, Philadelphia, Pa, USA, 1994.

[22] E. Carlos, C. E. de Souza, A. Trofino, and K. A. Barbosa, "Modeindependent $H_{\infty}$ filters for Markovian jump linear systems," IEEE Transactions on Automatic Control, vol. 51, no. 11, pp. 18371841, 2006. 


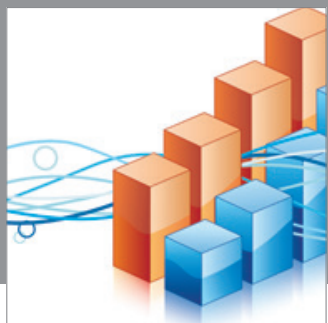

Advances in

Operations Research

mansans

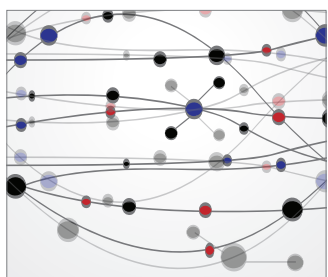

The Scientific World Journal
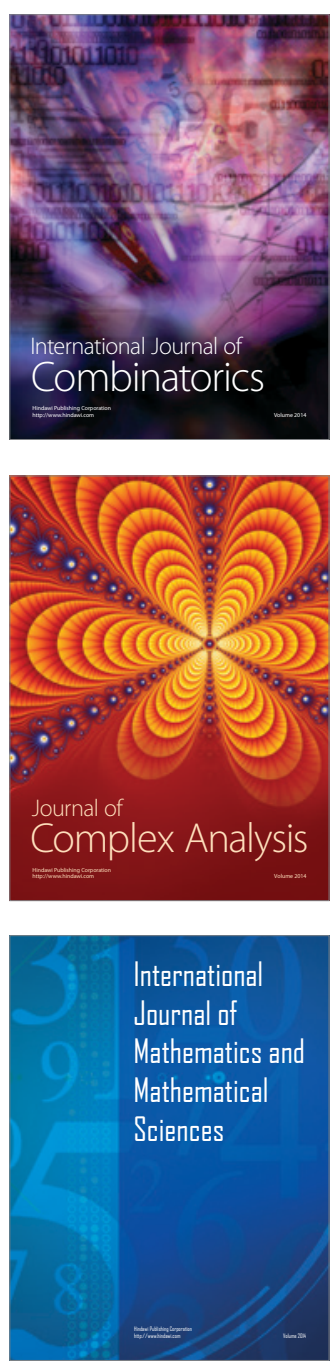
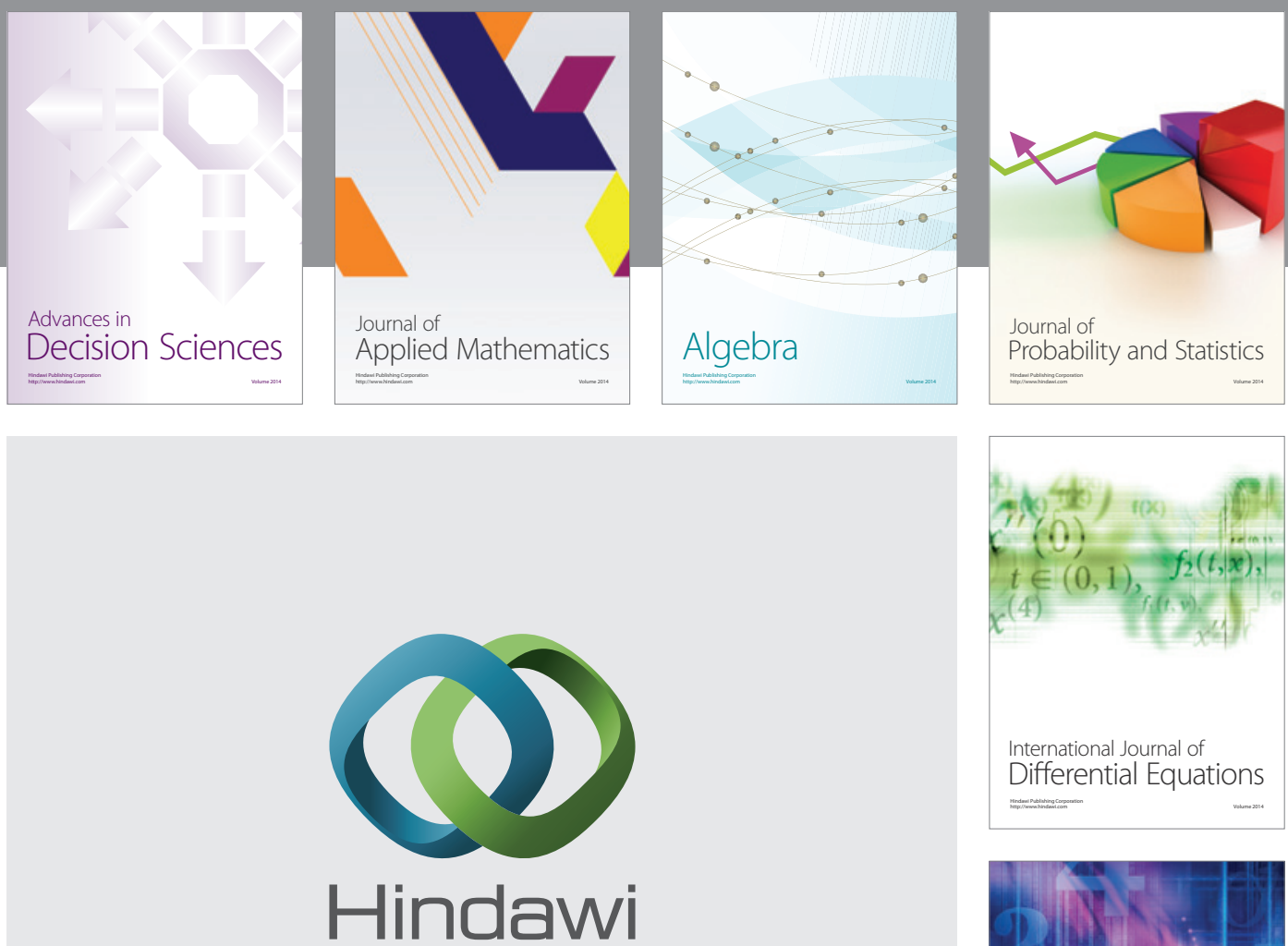

Submit your manuscripts at http://www.hindawi.com
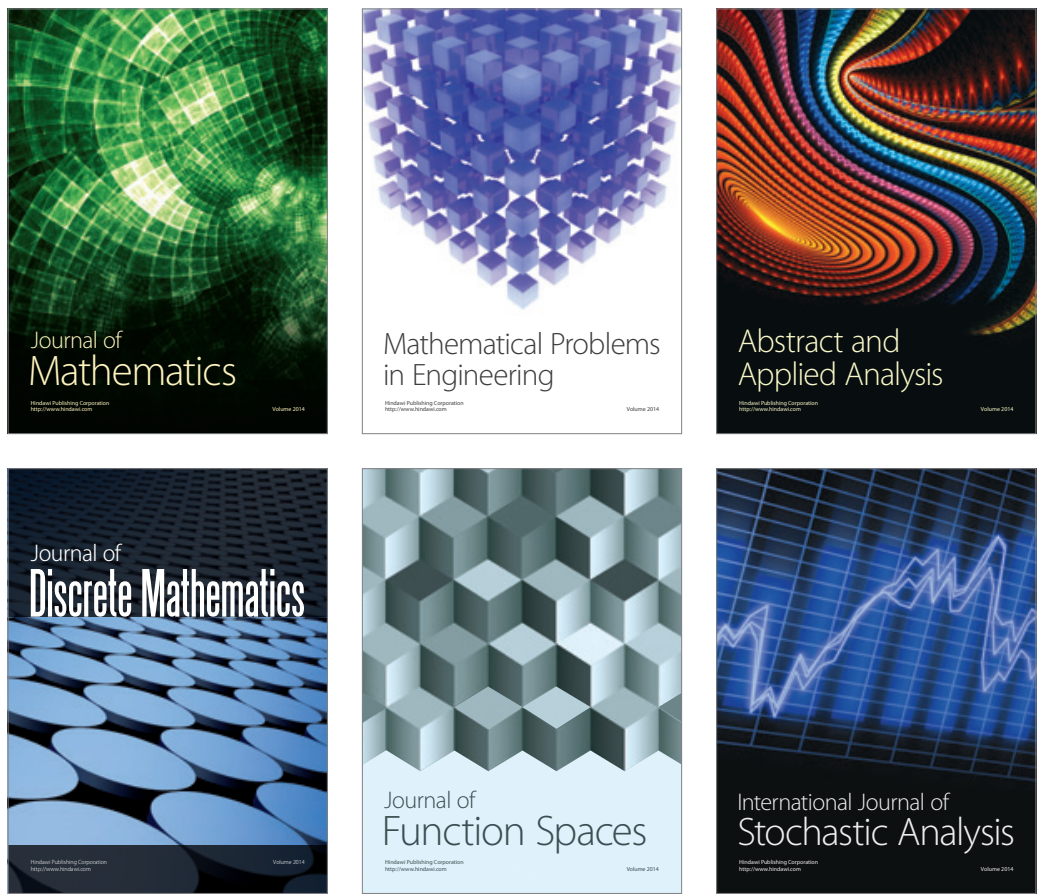

Journal of

Function Spaces

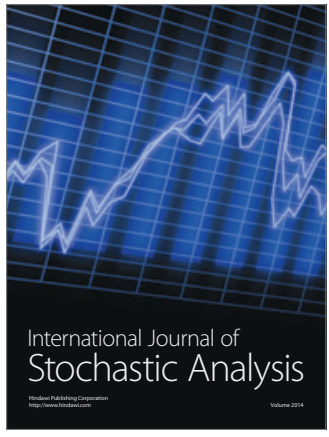

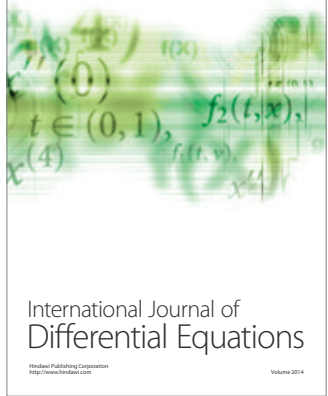
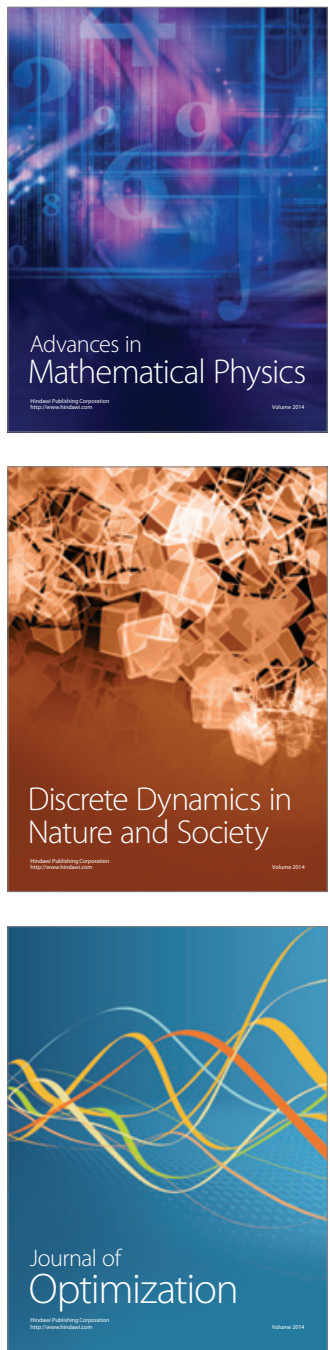